\title{
Development of a high-precision straightness measuring system with DVD pick-up head
}

\author{
Kuang-Chao Fan ${ }^{1,4}$, Chih-Liang Chu ${ }^{2}$, Jarn-Lien Liao ${ }^{1}$ and \\ Jong-I Mou ${ }^{3}$ \\ ${ }^{1}$ Department of Mechanical Engineering, National Taiwan University, Taipei, Taiwan, \\ Republic of China \\ ${ }^{2}$ Department of Mechanical Engineering, Southern Taiwan University of Technology, Tainan, \\ Taiwan, Republic of China \\ ${ }^{3}$ Department of Industrial Engineering, Arizona State University, Tempe, Arizona, USA \\ E-mail: fan@ccms.ntu.edu.tw
}

Received 12 September 2002, accepted for publication 29 October 2002

Published 26 November 2002

Online at stacks.iop.org/MST/14/47

\begin{abstract}
A low-cost and high-precision straightness measurement system using a DVD pick-up head has been developed in this research. By removing its objective lens the pick-up head of a commercial DVD player possesses excellent properties of stable laser power, collimated laser beam, circular Gaussian distribution and sensitive photodetectors. A moving knife-edge piece is placed between the pick-up head and a plane mirror. According to the knife-edge principle, the non-shaded part of the laser beam will be reflected back and focused onto the four-quadrant photodetector of the pick-up head. The four-quadrant signals are added to measure the returned laser power. Theoretical analysis based on the circular Gaussian distribution theory and regression method can attain the straightness error of the knife-edge while it moves along the beam direction. This low-cost system can apply to the straightness measurement of precision coordinate measuring machines and NC machine tools. Experimental results showed the achieved accuracy is better than $\pm 0.2 \mu \mathrm{m}$ within the measuring range of $200 \mu \mathrm{m}$.
\end{abstract}

Keywords: DVD pick-up head, straightness measurement, knife-edge principle

(Some figures in this article are in colour only in the electronic version)

\section{Introduction}

As technology advances, the requirement for parts and products quality becomes more and more stringent. There are numerous standard metrology terms that can be used to depict the quality of a part or product. The term 'straightness error' is generally used to refer to many aspects of engineering quality, such as workpiece straightness, motion straightness, etc.

Optical measurement of straightness errors has been widely used in the field of engineering metrology. Two kinds of methods are commonly adopted. One uses the optical axis

4 Author to whom any correspondence should be addressed. as an invisible reference line, a straightness datum that can be established by means of various optical accessories, such as the cross-hair reticle. These instruments include autocollimators, alignment telescopes and optical theodolites $[1,2]$. The other method employs a He-Ne laser beam to generate a visible reference line, which has the properties of small beam drift, small beam divergence and high intensity. The beam centre was taken as the reference point whose position was examined using a four-quadrant detector or a CCD camera. A resolution of $1 \mu \mathrm{m}$ was achieved [2-4].

Many other methods have been developed with high alignment accuracy such as the polarimetry method [5], the 
polarizing method [6], the optical compensation method [7], the Zeeman laser interferometer method [8], and the optical self-adapting principle [9] to eliminate the measuring error resulted from beam drift. Recently, the laser diode was adopted for straightness measurement because of its small size, low cost, and low power requirement [10]. An earlier study that integrated a laser diode with fibre optical and modulation techniques developed a laser straightness measurement system that automatically compensates for disturbing lights, circuit drift, and thermal related beam drift. The achieved measurement accuracy is $0.3 \mu \mathrm{m}$ [11].

Although straightness error assessment has become an important quality assurance requirement, the cost for a precision autocollimator is quite expensive. The goal of this study is to develop a low-cost yet high-precision laser straightness measuring system.

Since the first CD (compact disc) was commercialized in 1982, the laser-based data acquisition technology has continuously progressed. Nowadays, the development of the optical pick-up head embedded in CD-ROM or DVDROM is quite mature. The well developed optical and circuit design, as well as the focusing characteristics, is well suited for optical metrology applications at low cost. Several systems have been developed for collimators [12], for profile measurements [13, 14], and for confocal compact scanning optical microscopes [15].

The most common laser output mode is $\mathrm{TEM}_{00}$ at which the laser intensity possesses Gaussian distribution. The modern DVD disc contains high-density data, therefore high laser spot quality is required for accurately acquiring the data stored on the fast-spinning disc. The laser beam must have stable power, circular Gaussian distribution, good parallelism, and small spot size. In this study, the aforementioned excellent laser characteristics are integrated with a knife-edge image analysis method to develop a laser straightness measuring system.

The technology of single knife-edge scanning is well developed and has been successfully applied in the development of various optical instruments. Firester [16] used a PZT to move a knife-edge in scanning a spot size less than $1 \mu \mathrm{m}$. By comparing with the measured displacement using an interferometer, the achieved accuracy is $\lambda / 8$. Khosrofian [17] used the knife-edge method to scan a He-Ne laser spot and a least-square linear regression method to analyse the light intensity data. The difference between the scanned and actual spot diameters is less than $0.05 \%$. Cronin [18] set up a surgical knife with a skew surface on a fast-moving scanning stage to characterize its mechanical frequency response.

From the related studies conducted in the past, we conclude that it will be cost effective to develop a precision straightness measurement system by adopting the commercially available DVD pick-up head and its embedded components such as laser diode, grating, beam splitter, fourquadrant photodetector, collimating lens, etc.

\section{Measurement principle}

\subsection{Pick-up head}

2.1.1. Principle and modification of DVD pick-up head. The DVD pick-up head uses a laser diode to produce optical light.

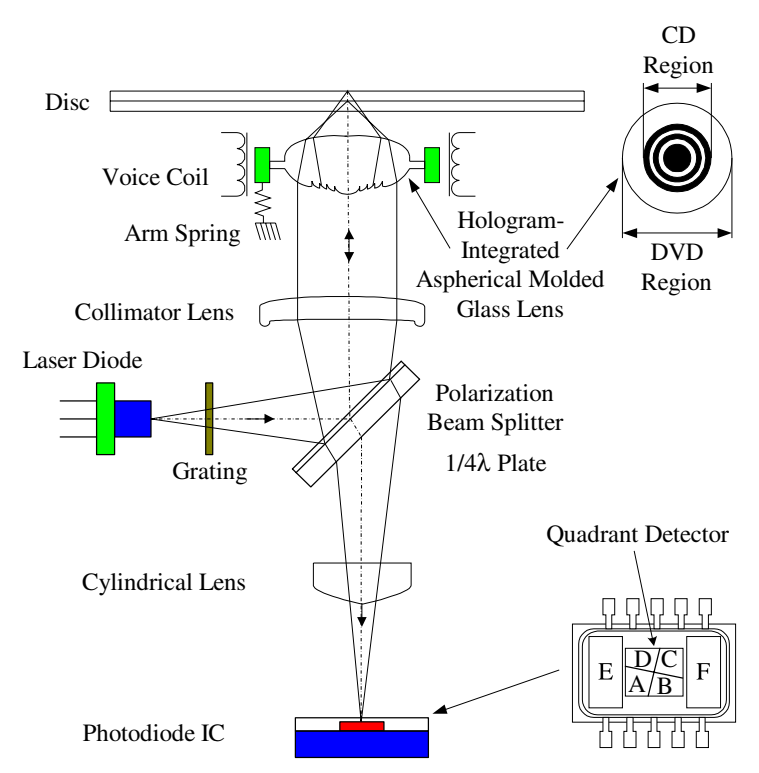

Figure 1. Structure of a DVD/CD pick-up head.

Passing through a grating the light diffracts into three beams, as shown in figure 1. These beams pass through a polarized beam splitter, a quarter wave plate, and a collimator lens to transform the laser beams into a collimated beam. The collimated laser beam is then passed through a holographic Fresnel lens that has concentric circular grooves with interval spacing of tens to hundreds of micrometre. The original collimated beam is then separated into two parts and focused to dual focal points, one for reading DVD data and the other for reading CD data.

The beams then reflect back along the original path and pass through the same collimator lens, beam splitter, and a cylindrical lens, and finally project onto the four-quadrant photodiode, which outputs a focus error signal (FES) according to the beam spot distribution among four quadrants. After proper signal processing, the FES is used to drive the voice coil motor (VCM) to shift the objective lens until the focal point is set on the disc surface. The pick-up head then acquires the stored data by transforming the FES into digital signals.

A well-designed DVD pick-up head should have a stable laser power. The energy intensity is generally assumed to have Gaussian distribution. To ensure the quality, the laser requires good parallelism, circular beam shape, and $\mathrm{TEM}_{00}$ output mode.

In this study, a Sony KHM-210AAA DVD pick-up head with $650 \mathrm{~nm}$ wavelength [19] is modified by removing the objective lens and the VCM, and outputs with a collimated laser beam, as shown in figure 2. A knife-edge piece (or mask) and a plane mirror are added to the system. The originally embedded four-quadrant photodiode is used as a light intensity detector. To ensure that the laser power will not fluctuate with ambient temperature, a specially designed power supply in association with an automatic power control (APC) feedback circuit is used to control the laser output power stability. Vertical movement of the knife-edge will block a part of the beam area projected onto the mirror and the remaining beam will be received by the four-quadrant photodiode. Although such a setup can induce some diffraction effect at the knife-edge if the mirror is placed quite a distance away (far field), say more than $10 \mathrm{~cm}$, only the main beam of the zeroth order will emit to the mirror. 


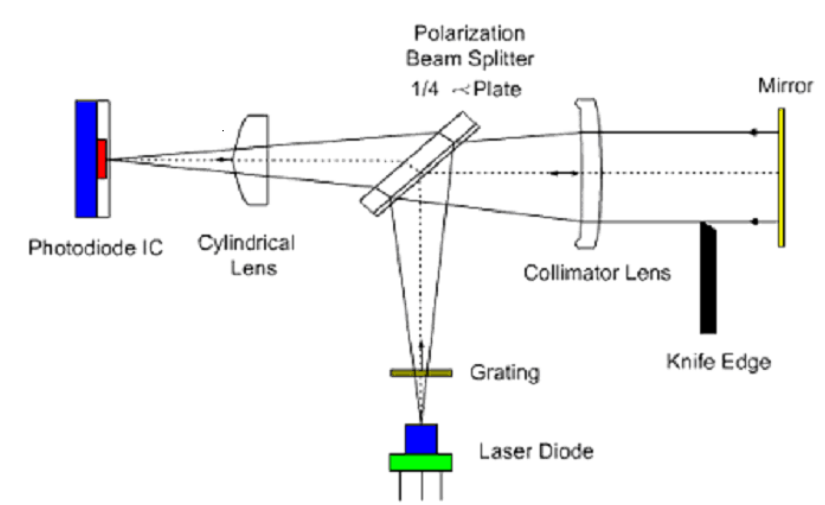

Figure 2. The knife-edge displacement measurement system.

2.1.2. Four-quadrant photodetector. The photodetector transforms the detected light intensity signal to an electrical current signal and with proper resistance the electrical current signal can then be converted to voltage. According to the voltage intensity, the photodetector interpolates the position where light strikes. With a four-quadrant photodetector the $X$ and $Y$ coordinates and corresponding voltages can be expressed as follows:

$$
\begin{aligned}
& X=K \frac{\left(V_{1}+V_{4}\right)-\left(V_{2}+V_{3}\right)}{V_{s}} \\
& Y=K \frac{\left(V_{1}+V_{2}\right)-\left(V_{3}+V_{4}\right)}{V_{s}}
\end{aligned}
$$

where $V_{i}=$ voltage for each quadrant, $i=1, \ldots, 4, K=$ constant and $V_{s}=$ sum of all four quadrant voltages $=$ $V_{1}+V_{2}+V_{3}+V_{4}$.

In this study, the four-quadrant photodetector embedded in the pick-up head is used only to sense the total light intensity $\left(V_{s}\right)$ that is unblocked by the knife-edge, as shown in figure 2 . When $V_{s}$ is zero, the reflected light is totally blocked by the knife-edge. The displacement of the knife-edge less the beam diameter corresponds to a certain $V_{s}$. In the following, we prove that this relationship obeys the law of Gaussian distribution.

\subsection{Laser beam scanning principle}

2.2.1. Laser beam intensity distribution. If the laser chamber is in lateral electromagnetic resonant $\left(\mathrm{TEM}_{00}\right)$ mode, the generated beam intensity will have Gaussian distribution and is denoted a Gaussian beam. As can be seen from figure 3, its electrical field can be expressed as

$$
\begin{aligned}
E(r, z) & =\underbrace{E_{0} \frac{W_{0}}{W(z)} \times \exp \left(-\frac{r^{2}}{W^{2}(z)}\right)}_{\mathrm{A}} \\
\times & \underbrace{\exp \left\{-\mathrm{j}\left[k z-\tan \left(\frac{z}{z_{R}}\right)\right]\right\}}_{\mathrm{B}} \times \underbrace{\exp \left[-\mathrm{j} k \frac{r^{2}}{2 R(z)}\right]}_{\mathrm{C}}
\end{aligned}
$$

In equation (3), term $\mathrm{A}$ is the amplitude factor that is a function of $r=\left(x^{2}+y^{2}\right)^{1 / 2}$, term $\mathrm{B}$ is the phase change relationship of the light wave along the longitudinal (z) direction and term $\mathrm{C}$ is the phase change relationship of the light wave along the radial $(r)$ direction. $W_{0}$ is the beam $(\mathrm{x}, \mathrm{y})$

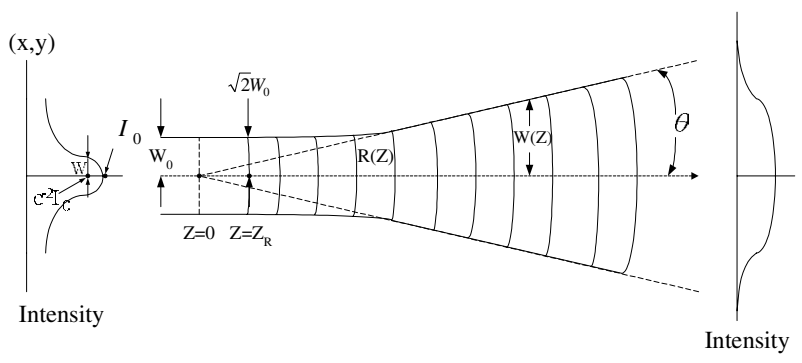

Figure 3. Gaussian laser beam intensity distribution characteristic.

waist radius when the wavefront curvature radius $R(0) \rightarrow \infty$ and the wave front curvature becomes a plane with a smallest beam diameter. $W(z)$ and $R(z)$ are the radii of the spot size and the wave front curvature at a distance $z$ from the waist, respectively. When $k=2 \pi / \lambda$ and setting the origin $(z=0)$ at the waist, we have

$$
\begin{gathered}
W(z)=W_{0}\left[1+\left(\frac{\lambda z}{\pi W_{0}^{2}}\right)^{2}\right]^{1 / 2}=W_{0}\left[1+\left(\frac{z}{z_{R}}\right)^{2}\right]^{1 / 2} \\
R(z)=z\left[1+\left(\frac{\pi W_{0}^{2}}{\lambda z}\right)^{2}\right]=z\left[1+\left(\frac{z_{R}}{z}\right)^{2}\right]
\end{gathered}
$$

where $z_{R}=\pi W_{0}^{2} / \lambda$ is defined as the Rayleigh range that is located in a plane with a standoff distance $z=z_{R}$ from the waist when the cross sectional area of the beam spot is exactly twice the waist area $\left[W\left(z_{R}\right)=\sqrt{2} W_{0}\right]$ and the wave front curvature radius $R$ is at its minimum.

When the beam propagating distance $z \gg z_{R}, R \approx z$ and $W \approx \lambda z / \pi W_{0}$, the Gaussian beam appears as a light emitted from a point at waist. Its divergence angle can be expressed as follows:

$$
\theta=\frac{\mathrm{d} W(z)}{\mathrm{d} z}=\frac{W_{0}}{z_{R}}=\frac{\lambda}{\pi W_{0}} .
$$

The Gaussian beam characteristics are determined by $W_{0}$ and $\lambda$. Since the electric field for the light wave changes quickly, the light intensity is frequently used in metrology applications. The light intensity distribution can be derived as the product of equation (3) and its complex conjugate, and expressed in Cartesian coordinate as follows:

$$
I=E \cdot E^{*}=I_{0} \exp \left\{\frac{-2\left[\left(x-x_{0}\right)^{2}+\left(y-y_{0}\right)^{2}\right]}{W^{2}}\right\}
$$

where $x_{0}, y_{0}$ is the beam centre, $I_{0}=I_{\max }$ is the light intensity at beam centre and $W$ is the cross sectional radius of the Gaussian beam.

2.2.2. Mathematical model of knife-edge scanning. If the knife-edge scanning across the beam is along the $X$-axis, as shown in figure 4 , the sensed light intensity of the unblocked laser beam can be expressed as

$$
\begin{aligned}
& S\left(x_{a}\right)=\int_{-\infty}^{\infty} \int_{x_{a}}^{\infty} I(x, y) \mathrm{d} x \mathrm{~d} y \\
& =\int_{-\infty}^{\infty} \int_{x_{a}}^{\infty} I_{0} \exp \left\{\frac{-2\left[\left(x-x_{0}\right)^{2}+\left(y-y_{0}\right)^{2}\right]}{W^{2}}\right\} \mathrm{d} x \mathrm{~d} y \\
& =I_{0}\left(\frac{\pi W^{2}}{2}\right)^{\frac{1}{2}} \int_{x_{a}}^{\infty} \exp \left\{\frac{-2\left(x-x_{0}\right)^{2}}{W^{2}}\right\} \mathrm{d} x
\end{aligned}
$$

where $x_{a}$ is the $X$ coordinate of the knife-edge. 


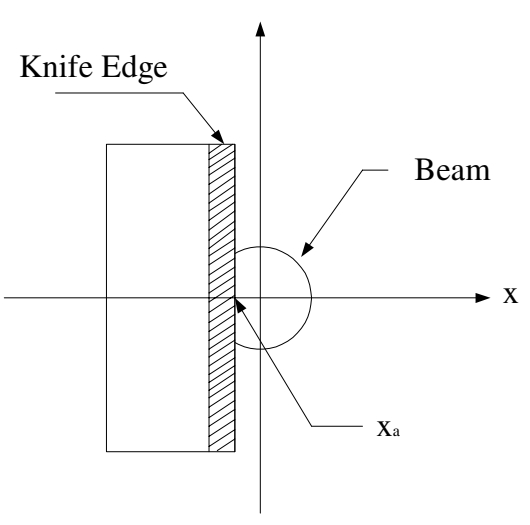

Figure 4. Knife-edge scanning across the beam along the $X$-axis.

The complete range of the laser beam intensity distribution curve is shown in figure 5(a). The difference between two intensity signals for position $x_{k}$ and $x_{k}+\Delta x$ can be expressed as follows:

$$
S_{A}\left(x_{k}\right)-S_{B}\left(x_{k}+\Delta x\right)=\int_{-\infty}^{\infty} \int_{x_{k}+\Delta x}^{x_{k}} I(x, y) \mathrm{d} x \mathrm{~d} y .
$$

The result of equation (9) is equivalent to the integral of the shaded area shown in figure 5(b).

A normalized laser intensity signal can be derived by dividing a partially blocked laser intensity signal by the totally unblocked laser intensity signal as follows:

$$
\bar{S}\left(x_{a}\right)=\frac{S\left(x_{a}\right)}{S(\infty)}=\left(\frac{2}{\pi \omega^{2}}\right)^{\frac{1}{2}} \int_{x_{a}}^{\infty} \exp \left[\frac{-2\left(x-x_{0}\right)}{\omega}\right] \mathrm{d} x .
$$

\subsection{Data process}

According to the theoretical analysis, the distribution of knife-edge scanned beam intensity versus position has the characteristic of an $S$-curve. Therefore, we adopt a nonlinear regression function, the logistic model [20], to transform parameters into a least-square regression function in matrix format. The logistic model expression is expressed as follows:

$$
Y_{i}=\frac{1}{1+\exp \left[-X_{i}\right]} .
$$

When $X_{i}$ approaches $\infty, 0$ or $-\infty, Y_{i}$ converges to 1 , 0.5 or 0 , respectively. A similar characteristic was depicted in figure 5(a). Equation (11) can be further modified by replacing the $-X_{i}$ term with $P_{i}(X)$ polynomial function as follows:

$$
Y_{i}=\frac{1}{1+\exp \left[P_{i}(X)\right]}
$$

where $P_{i}(X)=\beta_{0}+\beta_{1} X_{i, 1}+\beta_{2} X_{i, 2}^{2}+\cdots+\beta_{P-1} X_{i, p-1}^{p-1} ; Y_{i}$ is the measured intensity voltage; $X_{i, p-1}$ is the knife-edge displacement; $\beta_{0}, \beta_{1}, \beta_{2}, \ldots, \beta_{P-1}$ are regression variables.

By taking logarithm transformation on equation (11), the transformed polynomial regression model is expressed as

$$
\log \left(Y_{i}^{-1}-1\right)=\beta_{0}+\beta_{1} X_{i, 1}+\beta_{2} X_{i, 2}^{2}+\cdots+\beta_{P-1} X_{i, p-1}^{p-1} .
$$

Letting $V_{i}=\log \left(Y_{i}^{-1}-1\right), Z_{i, 1}=X_{i, 1}, Z_{i, 2}=X_{i, 2}^{2}, \ldots$ and $Z_{i, p-1}=X_{i, p-1}^{p-1}$, equation (13) can be rewritten as a linear regression model:

$$
V_{i}=\beta_{0}+\beta_{1} Z_{i, 1}+\beta_{2} Z_{i, 2}+\cdots+\beta_{p-1} Z_{i, p-1} .
$$

Equation (14) can be expressed in matrix form as

$$
\underset{n \times 1}{\boldsymbol{V}}=\underset{n \times p}{\boldsymbol{Z}} \underset{p \times 1}{\beta}
$$

where

$$
\begin{gathered}
\underset{n \times 1}{\boldsymbol{V}}=\left[\begin{array}{c}
V_{1} \\
V_{2} \\
\vdots \\
V_{n}
\end{array}\right] ; \\
\underset{n \times p}{\boldsymbol{Z}}=\left[\begin{array}{ccccc}
1 & Z_{11} & Z_{12} & \cdots & Z_{1, p-1} \\
1 & Z_{21} & Z_{22} & \cdots & Z_{2, p-1} \\
\vdots & \vdots & \vdots & \cdots & \vdots \\
1 & Z_{n 1} & Z_{n 2} & \cdots & Z_{n, p-1}
\end{array}\right] ; \\
\underset{p \times 1}{\beta}=\left[\begin{array}{c}
\beta_{0} \\
\beta_{1} \\
\vdots \\
\beta_{p-1}
\end{array}\right] .
\end{gathered}
$$

To determine the regression variable vector $\beta_{p \times 1}$, the standard least-square linear regression estimation method is adopted:



The least-square estimation value is

$$
\underset{p \times 1}{\beta}=\underset{p \times p}{\left(\boldsymbol{Z}^{T} \boldsymbol{Z}\right)} \underset{p \times 1}{\boldsymbol{Z}^{T}} \boldsymbol{V} .
$$

The least-square estimation value derived from equation (17) has the characteristics of unbiasedness, smallest deviation, unbiased estimation, consistency, and sufficiency. Thus, by substituting equation (17) into equation (11) we can derive a best-fitting curve and function.

\section{Measurement capability analysis and system setup}

After the developed system was designed and analysed, the DVD laser pick-up head went through a procedure of performance characterization and actual measurement validation.

\subsection{DVD laser beam analysis}

In order that the laser beam can be focused to the smallest spot size for high-density data acquisition, the laser must have stable output power, high parallelism, circular beam shape, and an output mode with basic $\mathrm{TEM}_{00}$ Gaussian distribution. To validate the DVD laser pick-up head had those characteristics, an experiment was conducted that used a CCD to capture images of the laser beam that passed through a polarizer for attenuating the beam intensity to avoid CCD saturation. The CCD-captured images were used to analyse the quality and stability of the laser spot. Figure 6 shows the CCD image of 

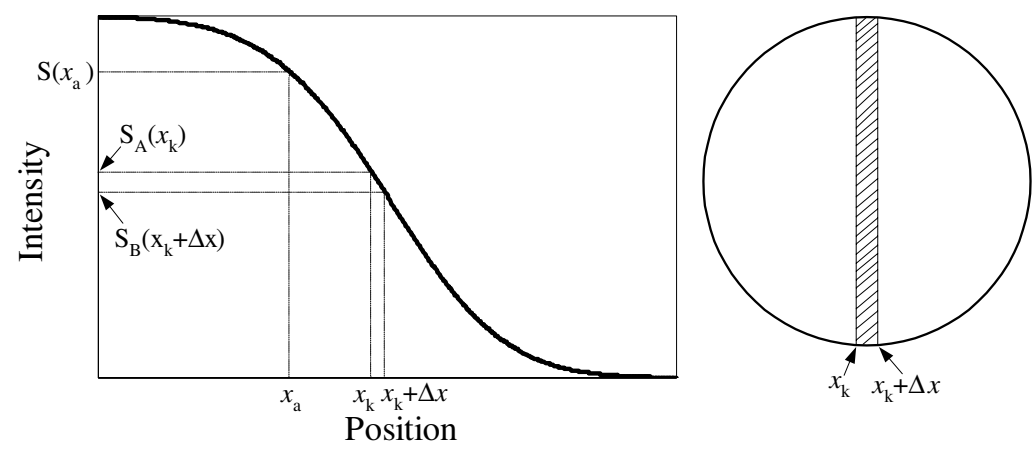

Figure 5. Gaussian laser beam intensity distribution curve.



Figure 6. Image of DVD laser beams.

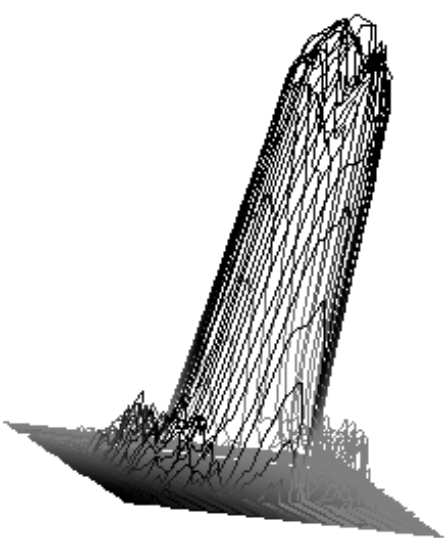

Figure 7. Distribution of grey-level values of DVD laser beam spot.

three captured laser beams, one primary beam for the main signal and two side beams for tilting signal: the light spot of the primary beam is circular, the images of the two secondary light beams are relatively blurred.

A 3D graphic package was used to plot the grey-level value for each pixel in the image. As shown in figure 7, the grey-level values show an excellent Gaussian distribution for the primary beam. As shown in figure 8 , the characteristic curves measured for the plane mirror at 1 and $2 \mathrm{~m}$ distance from the pick-up head are almost the same. Thus, the desired high parallelism is validated.

\subsection{The influence of laser power}

Similar to all other diodes, the laser diode output power is very sensitive to ambient temperature. Therefore, APC circuit is

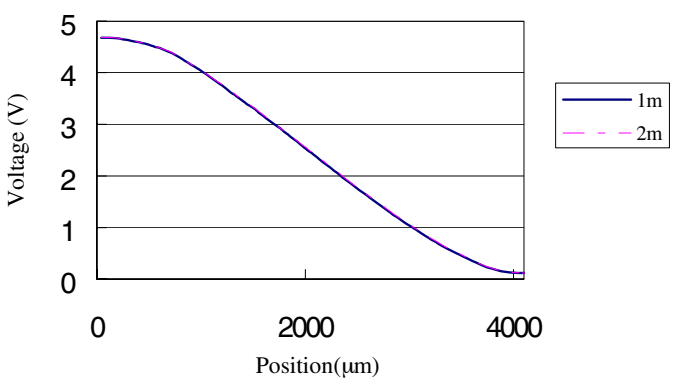

Figure 8. Knife-edge scanned intensity at different distances.

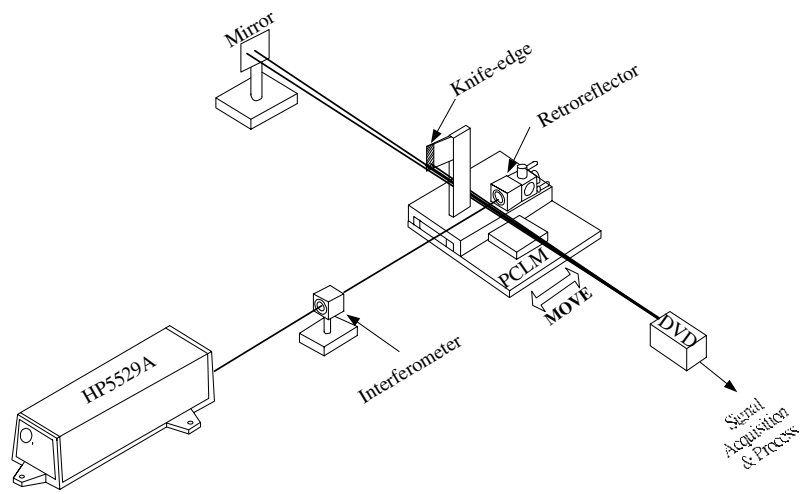

Figure 9. Setup for the knife-edge scanning measurement system.

commonly used to control the stability of laser power output. For feedback control, a photodetector is also commonly used to continuously monitor the laser diode output power. The laser diode is also very sensitive to current spike. Therefore, a slow-start protection device must be installed in the laser power supply circuit to avoid potential damage to the laser diode by sudden large current supply. A detailed description of the APC design and its performance test has been given in the authors' previous report [14]. The laser output power against operation time can be maintained constant with less than $1 \%$ fluctuation. To further improve the stability of the laser output power, a real-time normalization technique is developed to use the photodetector intensity signal output as reference to regulate the laser current supply. Therefore, the laser output power and photodetector output must have a linear relationship. 


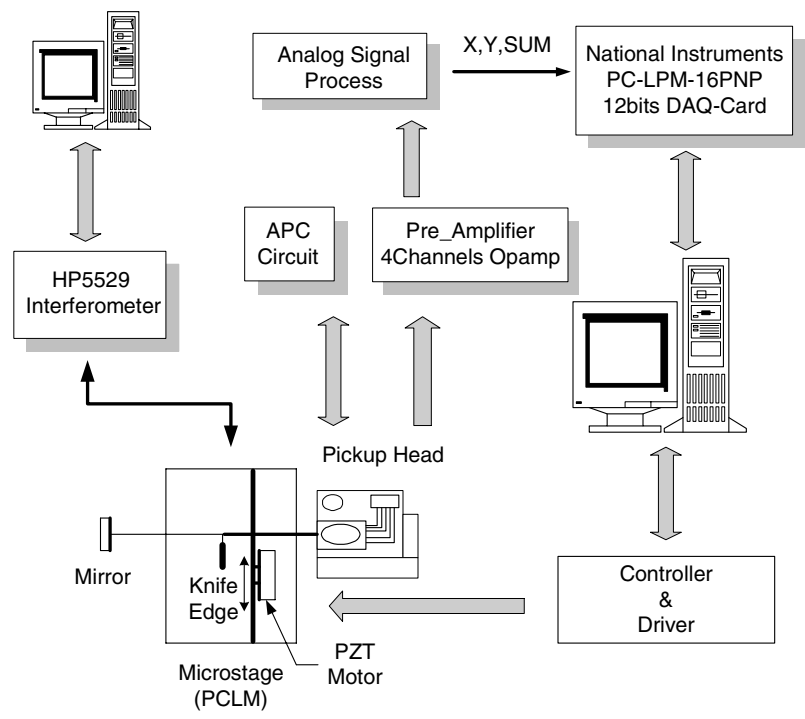

Figure 10. Setup for calibration of the measurement system.

Table 1. Calibrated repeatability.

\begin{tabular}{ll}
\hline Number & Standard deviation $\sigma(\mu \mathrm{m})$ \\
\hline 1 & 0.093512 \\
2 & 0.096272 \\
3 & 0.095526 \\
4 & 0.093215 \\
5 & 0.094235 \\
6 & 0.093871 \\
7 & 0.093545 \\
8 & 0.096366 \\
9 & 0.093591 \\
Average & 0.094 \\
\hline
\end{tabular}

\subsection{Setup and calibration of DVD laser straightness measurement system}

The setup of the developed DVD laser measurement system is shown in figure 9. A knife-edge was fastened on a precision micro-linear stage (PCLM-SP4, made by Anorad Co. USA). The motion of the PCLM was measured by a HP 5229A laser interferometer. The distance between the DVD pick-up head and the reflection mirror is larger than $1 \mathrm{~m}$. A National Instruments PC-LPM-16PNP data acquisition interface was used to collect reflected beam intensity and stage displacement signals. The measurement system configuration is shown in figure 10.

Figure 11 shows a best-fit relationship plot for the Gaussian beam distribution and stage displacement derived from a third-order logistic model.

The fitting accuracy can be evaluated by investigating the coefficient of correlation, $R . \quad R^{2}$ is defined as the coefficient of determination. The fitting accuracy increases as $R^{2}$ approaches 1 . The experimental data of figure 11 yield $R^{2}=0.999863$ which validates the fitting accuracy. From the plot of fitting residuals in figure 12 it can be seen that the accuracy of the developed system is better than $\pm 3.5 \mu \mathrm{m}$ within a range of $2100 \mu \mathrm{m}$.

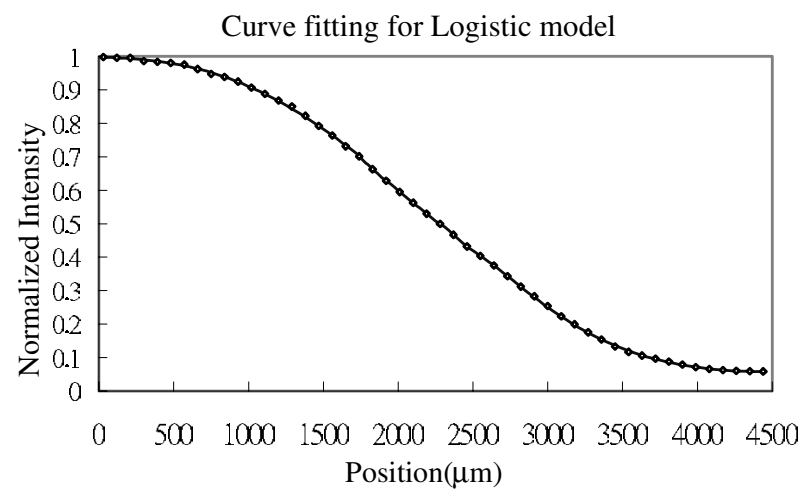

Figure 11. Laser intensity distribution plot; $\bullet$ measured data; - - best-fitted curve.

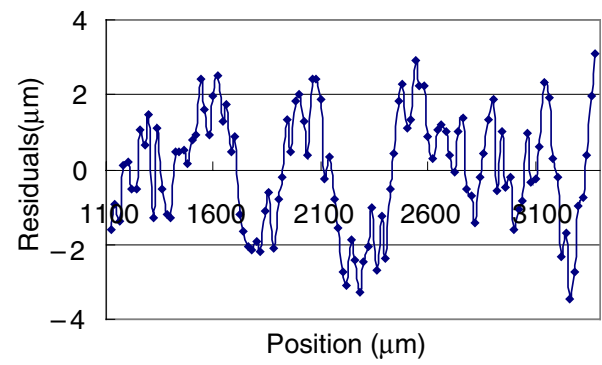

Figure 12. Fitting residual plot.

\subsection{Influence of laser beam diameter on system accuracy}

According to the data shown in figure 12, the system accuracy is still insufficient for precision metrology application. To improve the sensitivity and accuracy of the developed system, an inverse use of a beam expander $\left(M=F_{1} / F_{2} \cong 20\right)$, called the 'beam reducer', was added in front of the DVD laser head to reduce the beam diameter from $4 \mathrm{~mm}$ to $200 \mu \mathrm{m}$, as shown in figure 13.

Similar to figure 12, the fitting residuals of the small-beam system were derived from the data best-fit process and are 


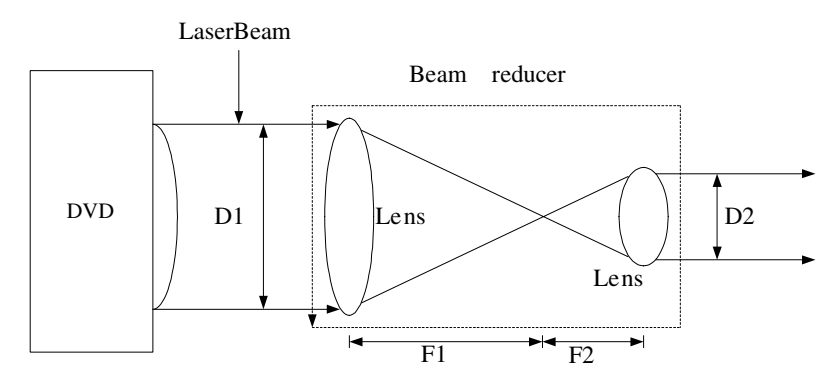

Figure 13. Reduction of the laser beam diameter.

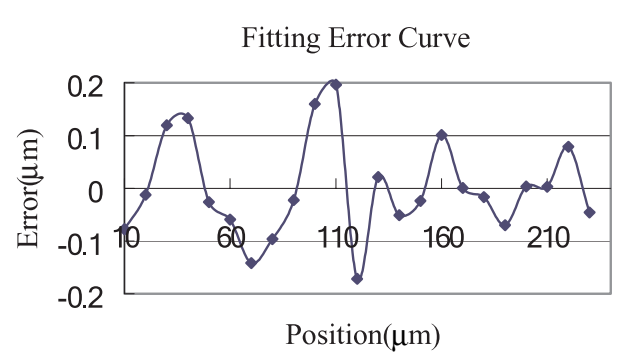

Figure 14. Fitting residual of the reduced beam.

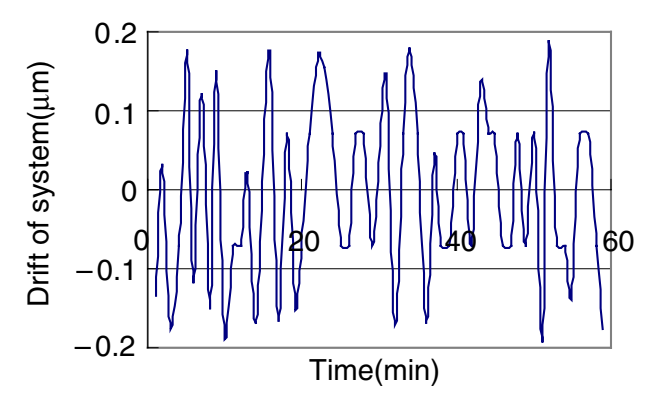

Figure 15. Intensity stability measurement results.

shown in figure 14. The modified system has significantly improved its accuracy to $\pm 0.2 \mu \mathrm{m}$ within a range of $200 \mu \mathrm{m}$.

\subsection{Repeatability test}

A repeatability analysis was then conducted to assess the system stability. Standard deviations of the fitting residual of nine repeated tests were calculated. Within a confidence level of $99.73 \%( \pm 3 \sigma)$, the developed system has uncertainty of $\pm 0.28 \mu \mathrm{m}$.

\subsection{Stability test}

With a knife-edge fixed near the middle of the beam spot, beam intensity was recorded for a one hour run. After substituting each beam intensity into the regression estimated curve equation, the corresponding estimated position drift was within $\pm 0.2 \mu \mathrm{m}$. As shown in figure 15, the system stability was assured. The figure also shows that the system position drift was within the regression-estimated position error.

\section{Applications}

The developed system was used to measure the straightness of a coordinate measuring machine (CMM) and a CNC milling machine.

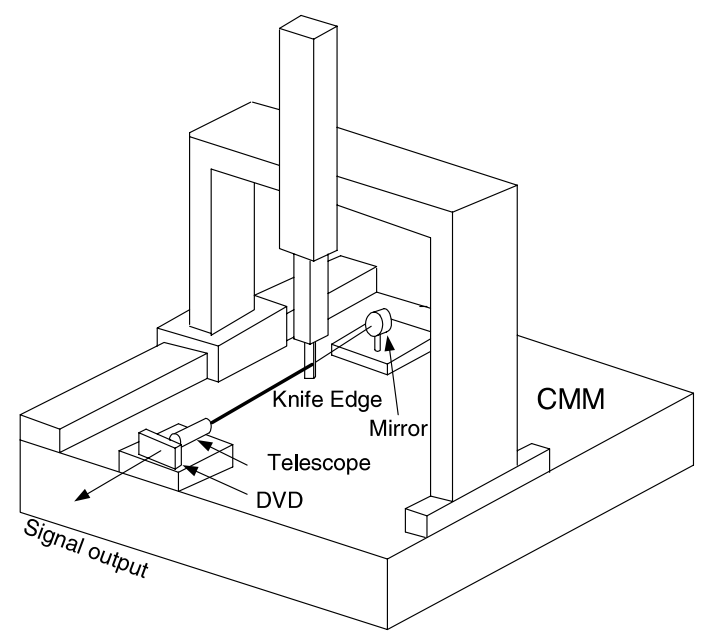

Figure 16. Straightness measurement setup on a CMM.

\subsection{Straightness measurement of a coordinate measuring machine}

The straightness measurement setup for a CMM is shown in figure 16. The DVD pick-up head and reflective mirror were set up on the granite table. The knife-edge was installed on the CMM probe spindle. The straightness error of the probe spindle motion parallel to the beam direction can be measured. Figure 17 shows the straightness error of the spindle, measured by the developed system and compared with an HP 5529 interferometer (straightness function with Wollaston prism), with forward and backward motion. A good consistency was achieved.

\subsection{Straightness measurement of a CNC milling machine}

The DVD pick-up head and reflective mirror were set up on the machine table. The knife-edge was installed on the tool spindle. The straightness error of the tool spindle motion along the beam direction can be measured. Figure 18 shows the straightness error of the spindle by the DVD system and the HP5529 straightness interferometer with forward and backward motions. Again, satisfactory results are obtained.

\section{Conclusions and discussion}

As the requirement of product quality and inspection accuracy become more stringent, the demands for a state-of-the-art optical measuring system increase. Adopting the technology of the commercially available DVD pick-up head, a lowcost yet high-precision laser straightness measurement system has been developed with $\pm 0.2 \mu \mathrm{m}$ accuracy and $\pm 0.28 \mu \mathrm{m}$ uncertainty $( \pm 3 \sigma)$ within a $200 \mu \mathrm{m}$ measuring range. The beam drift is about $\pm 0.2 \mu \mathrm{m}^{-1} \mathrm{~h}$. The tested piece or motion can be guaranteed to at least $2 \mathrm{~m}$.

A few factors have been identified for potential system instability: (1) electronic circuit instability, (2) thermal induced structure deformation and rigidity degradation, (3) laser beam drift, (4) air turbulence and ambient temperature fluctuation. The authors' previous research has proved that the electronic circuit instability can be removed by a modulation-demodulation technique and use of a digital 


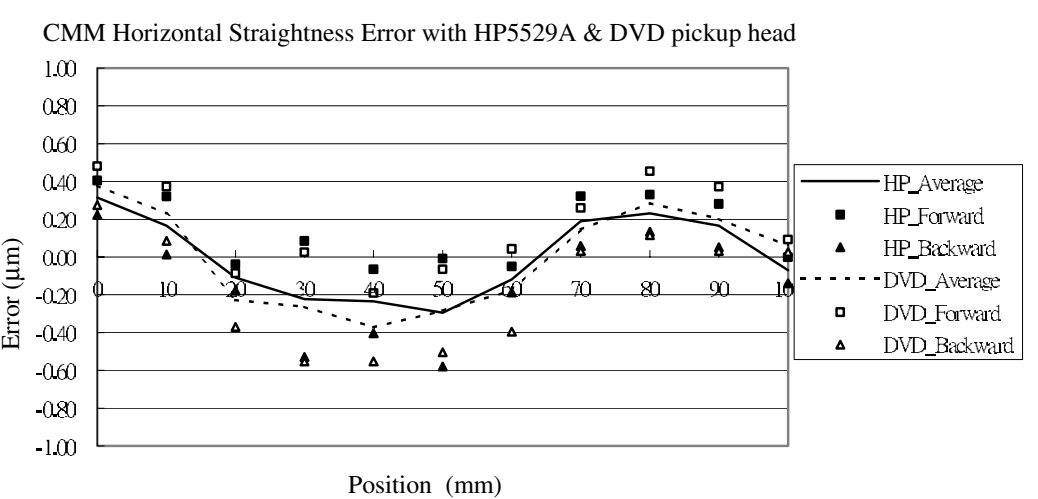

Figure 17. Straightness error of a CMM measured by DVD system and HP interferometer.



Figure 18. Straightness error of a milling machine measured by DVD system and HP interferometer.

signal processor [10]. The laser beam drift can also be reduced by inserting a fibre or monitored by a four-quadrant photodiode. The air turbulence and ambient temperature should be properly controlled in the testing environment. In addition, the measurement accuracy and robustness would be further improved if the beam diameter were further reduced to increase the sensitivity and signal-to-noise ratio.

\section{References}

[1] Greve J W and Wilson F W 1967 Handbook of Industrial Metrology (Englewood Cliffs, NJ: Prentice-Hall)

[2] Burdekin M 1980 Instrumentation and techniques for the evaluation of machine tool errors Technology of Machine Tools vol 5 (Livermore, CA: MTTF) section 9.7

[3] Weck M M 1980 Geometric and kinematic errors Technology of Machine Tools vol 5 (Livermore, CA: MTTF) section 9.12

[4] Chou et al C 1997 CCD based CMM geometrical error measurement using Fourier phase shift algorithm Int. J. Mach. Tool Manuf. 37 579-90

[5] King R J and Raine K W 1981 Polarimetry applied to alignment and angle measurement Opt. Eng. 201 39-43

[6] Ni J, Hiang P S and Wu S M 1992 A multi-degree-of-freedom measurement system for CMM geometric errors J. Eng. Ind. Trans. ASME 114 362-9

[7] Ye S G and Fan Y Z 1986 A new method to improve the alignment precision Proc. IMEKO TC, no 14 (Budapest, Hungary)

[8] Shimizu S, Lee H S and Imai N 1994 Simultaneous measuring method of the table motion errors in 6 degrees of freedom Int. J. Japan Soc. Prec. Eng. 28 273-4
[9] Guo J H, Cheng X G and Yin C Y 1997 Application of adaptive principle on laser straightness systems $J$. Tsinghua Univ. 37 21-3

[10] Fan K C and Zhao Y 2000 A Laser Straightness measurement system using optical fiber and modulation technique Int. J. Mach. Tool Manuf. 40 2073-81

[11] Sohn Y J, Kwon J H and Choe O S 1998 Portable autocollimators using the laser diode and the position sensitive detector Rev. Sci. Instrum. 69 402-5

[12] Armstrong T R and Fitzgerald M P 1992 An autocollimator based on the laser head of a compact disc player Meas. Sci. Technol. 3 1072-6

[13] Fan K C, Lin C Y and Shyu L H 2000 Development of a low-cost focusing probe for profile measurement Meas. Sci. Technol. 11 1-7

[14] Fan K C, Chu C L and Mou J I 2001 Development of a low-cost autofocusing probe for profile measurement Meas. Sci. Technol. 12 2137-46

[15] Benschop J and Rosmalen G V 1991 Confocal compact scanning optical microscope based on compact disc technology Appl. Opt. 30 1179-84

[16] Firester A H, Heller M E and Sheng P 1977 Knife-edge scanning measurements of subwavelength focused light beams Appl. Opt. 16 1971-4

[17] Khosrofian J M and Garetz B A 1983 Measurement of a Gaussian laser beam diameter through the direct inversion of knife-edge data Appl. Opt. 22 3406-10

[18] Cronin P J, Fekete P W, Arnison M R and Cogswell C J 2000 Characterization of an open-loop controlled scanning stage using a knife edge optical technique Rev. Sci. Instrum. 71 $118-23$

[19] Sony 2000 Specifications of Model KHM-210AAA

[20] Neter J, Wasserman W and Kutner M H 1990 Applied Linear Statistical Models: Regression, Analysis of Variance, and Experimental Designs (Homewood, IL: Irwin) 